\title{
The Elementary Proof of the Prime Number Theorem
}

\section{Joel Spencer and Ronald Graham}

L) rime numbers are the atoms of our mathematical universe. Euclid showed that there are infinitely many primes, but the subtleties of their distribution continue to fascinate mathematicians. Letting $\pi(n)$ denote the number of primes $p \leq n$, Gauss conjectured in the early nineteenth century that $\pi(n) \sim n / \ln (n)$. In 1896, this conjecture was proven independently by Jacques Hadamard and Charles de la Vallée-Poussin. Their proofs both used complex analysis. The search was then on for an "elementary proof" of this result. G. H. Hardy was doubtful that such a proof could be found, saying if one was found "that it is time for the books to be cast aside and for the theory to be rewritten."

But in the Spring of 1948 such a proof was found.

Almost immediately there was controversy. Was the proof attributable to Atle Selberg or was the proof attributable to Atle Selberg and Paul Erdős? For decades there seemed to be two mathematical camps with wildly different viewpoints. In the twenty-first century the controversy has finally subsided. Among previous discussions of the controversy, we mention particularly Goldfeld [1] (from which the previously mentioned quotation by Hardy is taken) and the book [3] of Paul Hoffman.

Ernst Straus was in a unique position to observe the beginnings of the controversy. He then held a position at the Institute for Advanced Study as a special assistant to Albert Einstein. (We believe Straus is the only person to have joint papers with Einstein and Erdős.) Straus had already worked a great deal with Erdős, and this work would continue throughout his life. Sometime in the early 1970s (we aren't sure of the exact dates), Straus wrote the account we present here. He did not want the notes to be published while the participants were still alive.

Ernst Straus was, for us and for many of his friends, a man of great wisdom. He certainly attempted in these notes to give as faithful an account of the events as he could. Whether he succeeded is a judgment for the reader to make. Certainly, he was far closer to Erdős than to Selberg. Let us be clear that we two authors both have an Erdös number of one, and our own associations with Erdös were long and profound. We feel that the Straus recollections are an important historic contribution. We also believe that the controversy itself sheds considerable light on the changing nature of mathematical research.

In November 2005, Atle Selberg was interviewed by Nils A. Baas and Christian F. Skau [4]. He recalled the events of 1948 with remarkable precision. We quote extensively from that account. Selberg had first shown that

$$
\sum_{p<x} \ln ^{2} p+\sum_{p q<x}(\ln p)(\ln q)=2 x \ln x+O(x)
$$

Erdős had heard about this through Paul Turán, and he wanted to see if he could use it to show that there exist prime numbers between $x$ and $x(1+\epsilon), \epsilon$ fixed and $x$ sufficiently large. The case $\epsilon=1$ is known as Chebyshev's Theorem. In 1933, at the age of 20, Erdös had found an elegant elementary proof of Chebyshev's Theorem, and this result catapulted him onto the world mathematical stage. It was immortalized with the doggerel

Chebyshev said it, and I say it again;

There is always a prime between $n$ and $2 n$

Clearly, Erdős would be very keen to find an elementary proof that worked for an arbitrary positive $\epsilon$. Selberg recalls:

I had the Prime Number Theorem in my thoughts, that was my goal based on [the previous] formula that I had 
obtained. I told [Erdös] that I did not mind that he try to do what he said he wanted to do, but I made some remarks that would discourage him.

Erdös succeeded in giving an elementary proof of the generalization of Cheybshev's Theorem to arbitray positive $\epsilon$. He showed some details of his proof to Selberg. Selberg continues:

So I told Erdös the next day that I could use his result to complete the proof, an elementary proof, of the Prime Number Theorem. [...] I really did not have in mind starting a collaboration with him.

At this point Selberg travelled to Syracuse where he was to take a position. This was the critical time. As Straus puts it, "Alas, something had gone wrong." The relationship between Selberg and Erdös had soured, never fully to be repaired. This story of great mathematical discovery becomes all too human. Selberg writes:

I started to hear from different sources that they only mentioned Erdös's name in connection with the elementary proof of the Prime Number Theorem, so I wrote a letter to Erdős.

Selberg proposes that they publish separately.

[Erdös] answered that he reckoned we should do as Hardy and Littlewood. But we had never made any agreement. In fact, we had really not had any collaboration. It was entirely by chance that he became involved in this - it was not my intention that he should have access to these things.

Paul Erdös and Atle Selberg were both giants of twentieth century mathematics. They (Erdös 1913-1996, Selberg 1917-2007) were of the same mathematical generation.

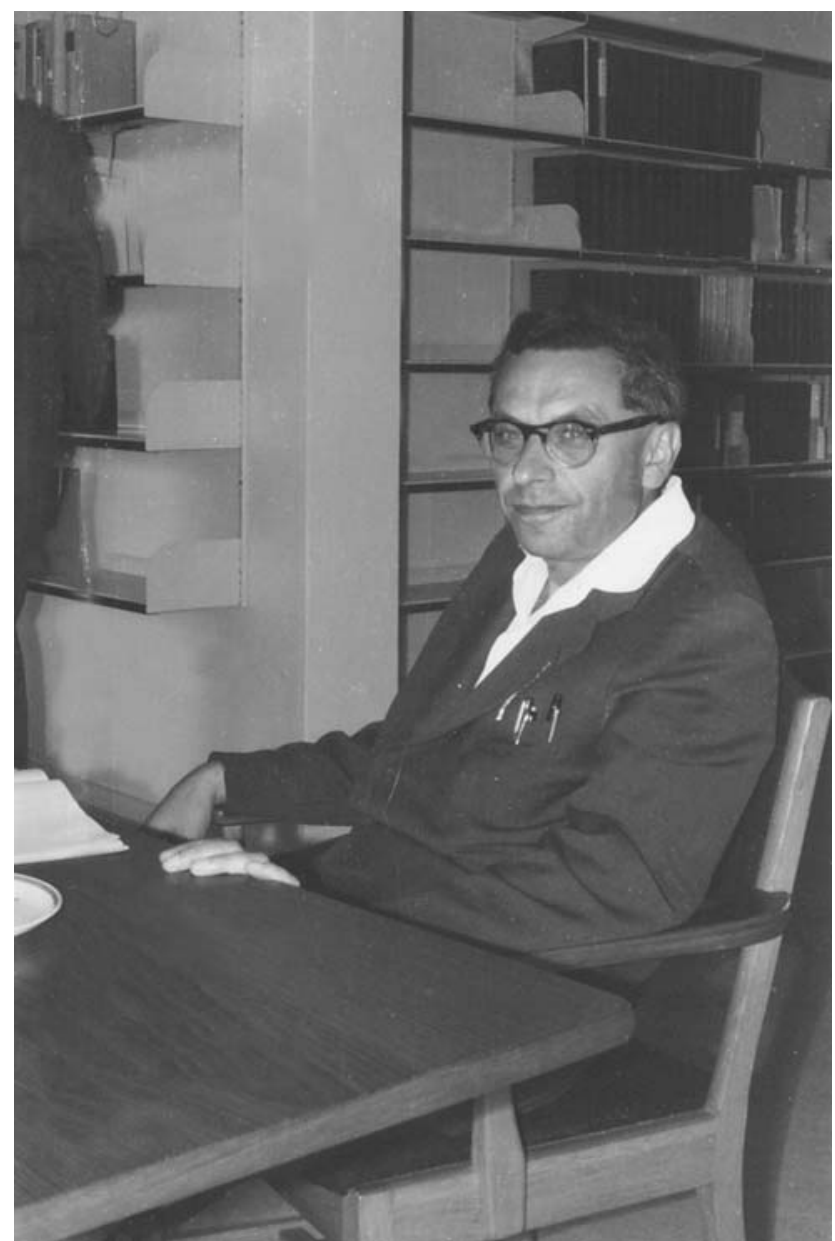

Figure I. Paul Erdős. 1962. Konrad Jacobs, photographer. Courtesy of the Archives of the Mathematisches Forschungsinstitut Oberwolfach.

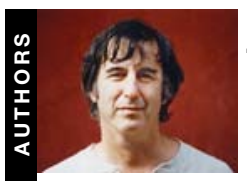

JOEL SPENCER is Professor of Mathematics and Computer Science at the Courant Institute (New York). His research centers on probabilistic methods (aka Erdős Magic) and probabilistic algorithms. He cofounded the journal Random Structures and Algorithms. He enjoys counting by nines with his grandson.

Courant Institute, New York, USA e-mail: spencer@courant.nyu.edu

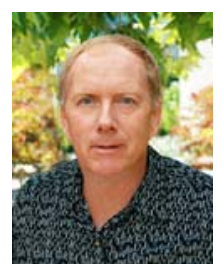

RONALD LEWIS GRAHAM "one of the principal architects of the rapid development worldwide of discrete mathematics in recent years," has held the presidencies of both The American Mathematical Society and the International Jugglers' Association, a feat that earned mention in Ripley's Believe It or Not. He is Irwin and Joan Jacobs Professor at the Department of Computer Science and Engineering of the University of California, San Diego (UCSD) and Chief Scientist at the California Institute for Telecommunication and Information Technology. His Erdős number is $1^{30}$.

Department of Computer Science
and Engineering
University of California San Diego
La Jolla, CA 92093-0404, USA
e-mail: graham@ucsd.edu

Department of Computer Science and Engineering University of California San Diego e-mail: graham@ucsd.edu 


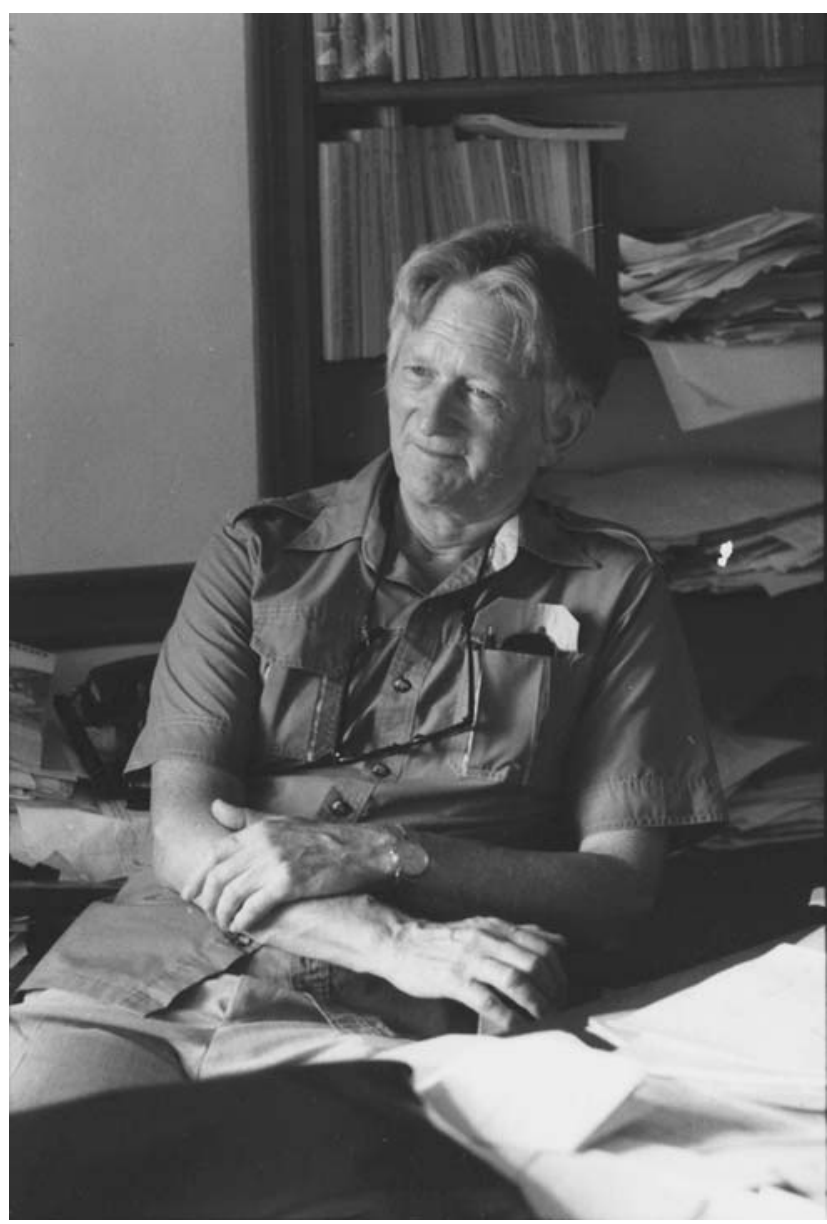

Figure 2. Atle Selberg, 1981. Hermann Landhoff, photographer. Courtesy of the Archives of the Institute for Advanced Study.

Both were prodigies. Erdös (so the stories go), at age five, realizing that 250 less than 100 is 150 below zero. Selberg, at age seven, showing that the difference between consecutive squares is an odd number. They were both winners of the prestigious Wolf Prize. But in (at least) two ways, however, their mathematical styles were antipodal.

For Paul Erdős, mathematics was a communal activity. Erdös had more coauthors, roughly five hundred by current counts, than any other mathematician in history. He constantly worked with a crowd of mathematicians surrounding him. For Atle Selberg, mathematical results were to be perfected in solitude and then to be brought forth. Nils Baas [5] writes "Selberg wanted to work on his own, penetrating the problems by his own and at his own pace." Selberg himself said, "I must say that I have never had any thought of collaborating with anybody. I have one joint paper, and that was with Chowla, but I must say that it was Chowla that first came to me with a question."

A deeper distinction is given in Tim Gower's beautiful essay "The Two Cultures of Mathematics." He explains [2]:

I mean the distinction between mathematicians who regard their central aim as being to solve problems, and those who are more concerned with building and understanding theories. [...] If you are unsure to which class you belong then consider the following two statements.

1. The point of solving problems is to understand mathematics better.

2. The point of understanding mathematics is to become better able to solve problems

For Gowers, Erdös is the ideal member of the problemsolving group, who would select the second statement without hesitation. Selberg would naturally be placed in the theory-builder category. Gowers continues,

It is that the subjects that appeal to theory-builders are, at the moment, much more fashionable than the ones that appeal to problem-solvers. Moreover, mathematicians in the theory-building areas often regard what they are doing as the central core (Atiyah uses this exact phrase) of mathematics, with subjects such as combinatorics thought of as peripheral and not particularly relevant to the main aims of mathematics.

We agree about the dichotomy, but not the animosity. Indeed, Gower's own well-deserved acclaim (including his receiving of the Fields Medal) from the entire mathematical community points to a less adversarial relationship. But it does seem to be a fair assessment of the mathematical landscape circa 1948, when the controversy began. We close with the words of Ernst Straus [6] himself, in a commemoration of Erdős's 70th birthday.

In our century, in which mathematics is so strongly dominated by "theory constructors" Erdös has remained the prince of problem solvers and the absolute monarch of problem posers. One of my friends - a great mathematician in his own right - complained to me that "Erdo"s only gives us corollaries of the great metatheorems which remain unformulated in the back of his mind." I think there is much truth to that observation, but I don't agree that it would have been either feasible or desirable for Erdös to stop producing corollaries and to concentrate on the formulation of his metatheorems. In many ways Paul Erdös is the Euler of our times. Just as the "special" problems that Euler solved pointed the way to analytic and algebraic number theory, topology, combinatorics, function spaces, etc.; so the methods and results of Erdős's work already let us see the outline of great new disciplines, such as combinatorial and probabilistic number theory, combinatorial geometry, probabilistic and transfinite combinatorics and graph theory, as well as many more yet to arise from his ideas.

Straus noted that Einstein chose physics over mathematics because he feared that one would waste one's powers in pursuing the many beautiful and attractive questions of mathematics without finding the central questions. He goes on,

Erdős has consistently and successfully violated every one of Einstein's prescriptions. He has succumbed to the seduction of every beautiful problem he has 
encountered - and a great many have succumbed to him. This just proves to me that in the search for truth there is room for Don Juans like Erdős and Sir Galahads like Einstein.

To modify slightly, the twentieth century had room for Don Juans like Paul Erdös and Sir Galahads like Atle Selberg.

\section{A Note on the Controversy}

\section{E. G. Straus}

Winter and Spring of 1948 were an exciting time in number theory at Princeton. Siegel was back from Germany, such relatively recent arrivals as A. Selberg, S. Chowla, and Paul Turán were at the Institute. Most stimulating for me was the presence of my - by then already long-term friend - Paul Erdös, not only in Princeton, but for several months as a guest in my house. This led to a number of interesting and stimulating discussions, including the now celebrated and still unsettled Erdös-Straus conjecture:

$$
\frac{4}{n}=\frac{1}{x}+\frac{1}{y}+\frac{1}{z}
$$

has a solution in positive integers $x, y, z$, for all integers $n>1$.

Turán, who was eager to catch up with the mathematical developments that had occurred during the war, talked with Selberg about his sieve method and his now famous inequality. He tried to talk Selberg into providing a seminar, showing the power of his inequality by giving an elementary proof of Dirichlet's Theorem on primes in arithmetic progressions; but Selberg, who was busy with other research and was also looking for a permanent academic position, declined. He suggested that Turán present the seminar, using the notes he had made for himself from his conversations with Selberg (perhaps even including some of Selberg's own notes).

This Turán did for a small group of us, including Chowla, Erdős and myself and, I think one or two others that I cannot recall with certainty. After the lecture in which Turán completed the elementary proof of Dirichlet's Theorem, there followed a brief discussion of the unexpected power of Selberg's inequality. Erdös said, "I think that you can also derive $p_{n+1} / p_{n} \rightarrow 1$ from this inequality. Some skepticism was expressed, also some question whether that result was more powerful than Dirichlet's theorem. In any case within an hour or two Erdös has discovered an ingenious derivation from Selberg's inequality. After presenting an outline of his proof to the Turán seminar group, Erdős met Selberg in the hall and told him that he could derive $p_{n+1} / p_{n} \rightarrow 1$ from Selberg's inequality. In retrospect, I am sorry that I did not commit Selberg's response to memory, but I remember its import exactly. He said, "You must have made a mistake, because with this result I can get an elementary proof of the Prime Number Theorem and I have convinced myself that my inequality is not powerful enough for that."

With that, excitement immediately reached a fevered pitch. Erdös and Selberg checked and rechecked every step of their respective proofs, and by about 10:00 p.m. they had convinced one another that the proofs were correct. An impromptu lecture was arranged in Fuld Hall. Since my wife was coming in from New York and was to arrive at Princeton Junction after the last shuttle train, the whole group (of nearly 50 people) was kind enough to wait until midnight. Then Selberg and Erdös in succession produced their results. There appeared to me to be an atmosphere of great joy, even elation, in the room. Many of us, including myself, had the feeling of attending an important historic event.

When we got home, too excited to go to sleep, Erdös and I discussed for some time the best way to spread the word. We both realized that at that time Erdös was far better known than Selberg and - at least in Erdös' mind the elementary proof was a direct outgrowth of Selberg's fundamental inequality, and Erdös' own contribution, although important, would not have been possible without that inequality. After lengthy discussion, we arrived at a formulation that Erdös used in the scores of postcards that he sent all over the world. I believe I remember the formulation verbatim "Using a fundamental inequality of Atle Selberg, Selberg and I have succeeded in giving an elementary proof of the Prime Number Theorem."

A second problem in Erdös' mind was how the results were to be published. We discussed this repeatedly with one another and with other mathematicians, and I can no longer say how much of it arose from Erdős, from myself, or from, say, Turán. However there was never any argument, just agreement that someone had made a good suggestion.

The result struck me as fair to all concerned. Erdös was to suggest that there would be back-to-back articles in the Annals. The first by Selberg alone, presenting his inequality and the derivation of Dirichlet's theorem from that inequality; the second jointly by Erdős and Selberg, presenting Erdös derivation of $p_{n+1} \sim p_{n}$ from Selberg's inequality and Selberg's completion of the proof of the Prime Number Theorem.

Thus anyone interested would obtain a clear picture of the respective contributions.

Alas, something had gone wrong, and when Selberg returned from Syracuse - which institution was interested in him and where he gave a lecture - he was no longer willing to work with Erdös at all.

It has always been a source of great surprise and regret to me that two such superb minds and admirable human beings, whom I both consider my friends - although Erdös is clearly a much closer friend than Selberg - have come to a permanent parting over a joint achievement that had been born with so much joy and hope. I have described Erdös' part in the matter and I am convinced that he did nothing intentional to hurt Selberg. I have already hinted at what may have been the source of unintentional pain, Erdös was much better known, certainly among contemporary American mathematicians, and even the careful wording of the postcards may not have prevented recipients from passing on the news as: "Have you heard what Erdős and some other guy have done?"

In fact I was told this story (I forget by whom), which may well not be true, but which illustrates my point: When 


\section{Ernst Straus}

Ernst Straus was born in 1922 in Munich. In 1933, after the Nazi rise to power, the Straus family emigrated to Palestine. He studied at the Hebrew University in Jerusalem. In 1941 he entered graduate school at Columbia University. He was the assistant to Albert Einstein from October 1944 through August 1948. He received his Ph.D. in 1948 from Columbia under the direction of F. J. Murray. He then accepted a position at UCLA, which he kept until his death of a heart attack in 1983.

During his Princeton years, Straus was strongly influenced by E. Artin and C. L. Siegel, and his major mathematical interests shifted from relativity theory to number theory. Throughout his life, Straus's mathematical interests continued to expand enormously and came to include geometry, convexity, combinatorics, group theory, and linear algebra, among other things.

In a memorial issue, ${ }^{*}$ from which the above information is taken, Straus's colleagues, David Cantor, Basil Gordon, Al Hales, and Murray Schacher, add the following tribute:

Ernst Straus was not only a great mathematician, but also a great human being. [...] His brilliance and enormous erudition in both humanities and science made a deep impression on all who were fortunate enough to know him. [...] This intellectual power was combined with a deep and radiant humanity which made Ernst truly beloved by his colleagues,

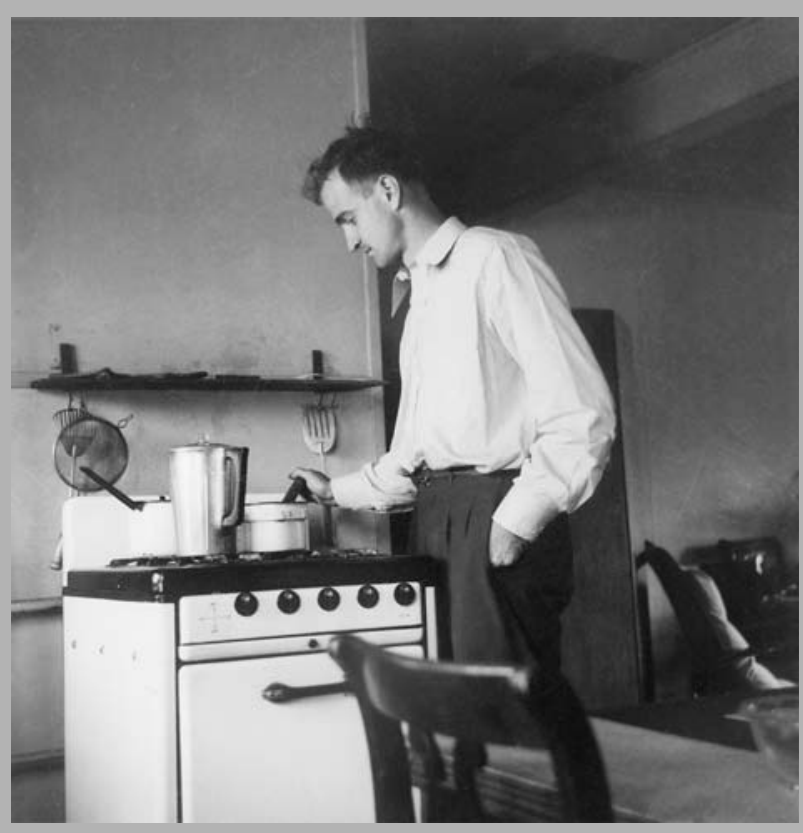

Ernst Straus, c. 1949, in housing at the Institute for Advanced Study. Photo courtesy of the Straus family.

students, and friends. [...] Men of such talent, uncompromising integrity, generosity, and gentleness are among the world's rarest and most precious treasures.
Selberg arrived in Syracuse he was met by a faculty member with the greeting: "Have you heard the exciting news of what Erdoss and some Scandanavian mathematician have just done?"

In any case, Selberg was still in his twenties and perhaps not yet sure of the great position he would fill in the mathematics of the twentieth century. In my mind more blame attaches to the actions and inactions of the older mathematicians who should have worked to conciliate, but instead served to exacerbate the situation. In my opinion the chief culprit was Hermann Weyl, who held strong esthetic views about the work of both Erdös and Selberg. The mathematics of Erdös went very much against Weyl's grain. This jumping from one challenging problem to the next, without first ascertaining its importance in the scheme of things, repelled Weyl, so that he never recognized the deep and important insights accumulated by Erdös as if by accident. It was Weyl who had vetoed the renewal of Erdös' grant at the Institute in the 1930s, throwing Erdös on the support of friends who were themselves hard pressed by the depression.

Selberg on the other hand was a proud discovery of Weyl on his first tour of Europe after the war. I remember with some awe that after Selberg presented his beautiful result on the positive density of zeroes of the Zeta-function on the critical line in Weyl's seminar, Weyl stood up to applaud - an event I have never seen before nor since in a mathematical seminar.

If one divides mathematicians - as I sometimes do for myself - into Euler types and Gauss types, then Erdös belongs to the former whereas Weyl and Selberg belong to the latter category.

It was Weyl who caused the Annals to reject Erdös' article and published only a version of Selberg that circumvented Erdös' contribution, without mentioning the vital part played by Erdös in the first elementary proof, or even in the discovery of the fact that such a proof was possible.

I think everyone of us who could have improved things and failed to do so has a share in the blame. Chowla was senior enough, but was probably too polite and deferential. Turán and I felt too closely associated with Erdös by longstanding friendships to be credible honest mediators. It may well be that nothing would have helped.

The elementary proof has so far not produced the exciting innovations in number theory that many of us expected to follow. So, what we witnessed in 1948, may in the course of time prove to have been a brilliant but somewhat incidental achievement without the historic significance it then appeared to have. My own inclination is to believe that it was the beginning of important new ideas not yet fully understood and that its importance will grow over the years.

* Ernst G. Straus 1922-1983. Pacific J. Mathematics, vol. 118 (1985) v-vi. 
In any case, the two mathematicians involved have justified our high expectations for their mathematical contributions.

\section{Postscript: A Note on the Effects of the Elementary Proof}

\section{Carl Pomerance, Dartmouth College}

The Riemann zeta function seems perfectly suited to study the natural numbers since intrinsic in this one function are both their additive and multiplicative structure. But maybe it is too perfect! Although knowing the Riemann Hypothesis and its generalizations would have remarkable consequences for the primes, this approach seems stymied. Instead, many recent great results about the distribution of primes and related problems have come about through the use of elementary and combinatorial ideas.

Thus, far from being an isolated intellectual challenge, the elementary proof of the prime number theorem was a signal that good ideas and strong tools are close at hand. We already had an inkling of this in Riemann's era when Chebyshev used combinatorial methods to show that there is a prime in $[n, 2 n]$ for every natural number $n$. And a century ago, the elementary proof of Brun, stating that most primes are not part of twin-prime pairs, opened the door for combinatorial sieve methods and their many glorious consequences.

Since the elementary proof, some of the most profound and exciting results in the field have had strong elementary and combinatorial leanings. After Roth used the (analytic) circle method to show that dense sets of integers must have 3-term arithmetic progressions, Szeméredi used an elementary (and very complicated) proof to generalize this to $k$-term arithmetic progressions. This result became an intrinsic tool in the recent Green-Tao proof that the set of primes contains arbitrarily long arithmetic progressions.

After Miller showed how a generalization of the Riemann Hypothesis allows a deterministic, polynomial-time procedure for recognizing primes, a few decades later, Agrawal, Kayal, and Saxena showed the same with completely elementary (and rigorous) methods.

I recall that both Erdős and Selberg were astonished when Maier used elementary tools to show the existence of unexpected irregularities in the distribution of the primes. This and subsequent results raise the tantalizing possibility of turning the tables and using elementary and combinatorial methods to say something about the distribution of zeta's zeros. For if these zeros tell us about the primes, why shouldn't the primes say something about them?

\section{OPEN ACCESS}

This article is distributed under the terms of the Creative Commons Attribution Noncommercial License which permits any noncommercial use, distribution, and reproduction in any medium, provided the original author(s) and source are credited.

\section{REFERENCES}

[1] Dorian Goldfeld, The Elementary Proof of the Prime Number Theorem: an Historical Perspective, in Number Theory (New York, 2003), 179-192, Springer, New York, 2004.

[2] Timothy Gowers, The Two Cultures of Mathematics, in Mathematics: Frontiers and Perspectives, V. Arnold, M. Atiyah, P. Lax, B. Mazur, eds, American Mathematical Society, 2000.

[3] Paul Hoffman, The Man who Loved Only Numbers: The story of Paul Erdős and the search for Mathematical Truth (New York, 1998), Hyperion.

[4] Nils A. Baas and Christian F. Skau, The Lord of the Numbers, Alte Selberg. On his Life and Mathematics. Bulletin of the American Mathematical Society, vol. 45 (2008), 617-649.

[5] Nils A. Baas, Speech at Atle Selberg Memorial at the Institute for Advanced Study, January 12, 2008.

[6] Ernst Straus, Paul Erdős at 70, Combinatorica, vol 3 (1983), 243246. 